Next, the team investigated the relationship between SVs and a number of phenotypes indicative of human health or disease risk, including mean arterial blood pressure, waist circumference and body mass index (BMI), among others. They found significant associations for 81 variable SVs and 43 deletion SVs in the Israeli cohort. Equivalent analyses of the Dutch samples showed that 40 of 117 associations in bacteria found in both cohorts were replicated, despite probable differences in genetic background and environment.

Again, the authors investigated the gene functions encoded by the SVs to obtain mechanistic insight into their associations with host health. A particularly informative example involved a $31 \mathrm{~kb}$ deletion SV in Anaerostipes hadrus that associated with lower weight, BMI and waist circumference and higher high-density lipoprotein cholesterol levels in the Israeli cohort. This SV and some of its associations (for example, lower weight and BMI) also replicated in the Dutch cohort.
The region was found to encode genes thought to be involved in transport and metabolism of sugar alcohols to butyrate, a short-chain fatty acid (SCFA), in a process that is energetically favourable for the bacteria. Furthermore, SCFAs (particularly butyrate) have been shown to have beneficial effects for the host, such as mitigating inflammatory disease and improving insulin sensitivity. The authors hypothesize that bacteria with this SV are therefore likely to have an improved symbiotic relationship with their host.

The authors note that causality, directionality and possible confounding were not investigated for the associations they describe. Nonetheless, the study demonstrates that scanning the microbiome for variable genomic regions that span multiple co-varying genes can help generate mechanistic hypotheses about the role of the microbiome in host health and disease.

Dorothy Clyde

ORIGINAL ARTICLE Zeevi, D. et al. Structural variation in the gut microbiome associates with host health. Nature 568, 43-48 (2019)

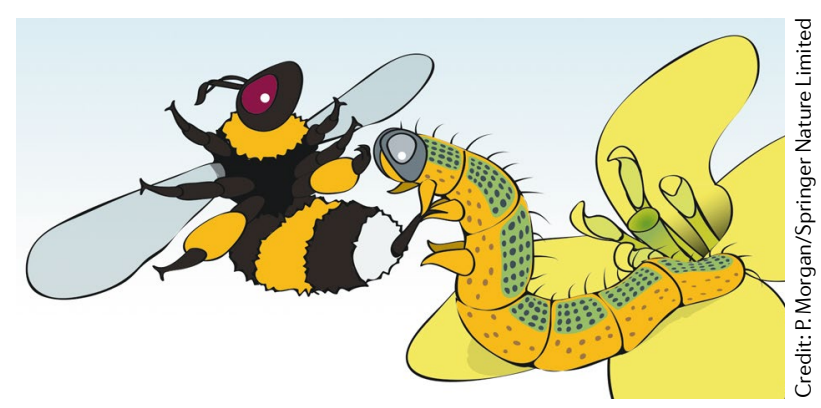

PLANT EVOLUTION

\section{Bug battles end in compromise}

Interaction with insects is known to drive evolution of plant diversity. However, the effects of different selective agents (such as pollinators and herbivores) tend to be studied in isolation, precluding a full understanding of how they interact to influence plant evolution. Now, a study in Science reveals that the combined presence of pollinators and herbivores alters the evolutionary trajectories of many plant traits and that plants can rapidly evolve to reduce trade-offs between different selective pressures.

Ramos and Schiestl performed experimental evolution on four different treatment groups of fast-cycling Brassica rapa plants: pollinators (Bombus terristris bumblebees) only; herbivores (Pieris brassicae caterpillars) only; both bees and caterpillars; and neither insect (the control group); with groups lacking bees pollinated by hand. After eight generations, a range of floral, defence and mating phenotypes were measured and compared between treatments. Even after this short timescale, significant differences were detected between groups. Notably, flowers that evolved in the presence of both bees and caterpillars were less attractive than those from the bee-only group but were more attractive than those from control plants. This finding suggests a trade-off between attracting pollinators and defending against herbivores, which probably involves the reallocation of resources. Consistently, bees and caterpillars also had an interactive effect on the levels of metabolites associated with plant defence. Effects on mating strategy were also observed: herbivory reduced herkogamy (the spatial separation between sexual organs) regardless of pollination method, and increased self-compatibility and autonomous mating in bee-pollinated plants. Importantly, this latter effect is probably indirect and a result of bees spending less time on caterpillar-infested plants, highlighting that evolutionary change can be elicited by altered pollinator behaviour.

The demonstration that crosstalk between selective agents leads to rapid evolution of plant traits underlines the need for a more integrative approach to investigating the evolution of diversity. As the authors note, such endeavours are particularly pertinent given the increasingly wide-ranging impact we humans have on our environment.

Dorothy Clyde

ORIGINAL ARTICLE Ramos, S. E. \& Schiestl, F. P. Rapid plant evolution driven by the interaction of pollination and herbivory. Science 364, 193-196 (2019) 